\title{
Télescope
}

Revue d'analyse comparée en adlministration publique

\section{Repères et Références}

Volume 19, numéro 1, hiver 2013

URI : https://id.erudit.org/iderudit/1017159ar

DOI : https://doi.org/10.7202/1017159ar

Aller au sommaire du numéro

Éditeur(s)

L'Observatoire de l'administration publique

ISSN

1929-3348 (numérique)

Découvrir la revue

Citer ce document

(2013). Repères et Références. Télescope, 19(1), 212-213.

https://doi.org/10.7202/1017159ar

Ce document est protégé par la loi sur le droit d'auteur. L'utilisation des services d'Érudit (y compris la reproduction) est assujettie à sa politique d'utilisation que vous pouvez consulter en ligne.

https://apropos.erudit.org/fr/usagers/politique-dutilisation/
Cet article est diffusé et préservé par Érudit.

Érudit est un consortium interuniversitaire sans but lucratif composé de l’Université de Montréal, l'Université Laval et l'Université du Québec à Montréal. Il a pour mission la promotion et la valorisation de la recherche. https://www.erudit.org/fr/ 


\section{Repères et Références}

Télescope a choisi des articles à lire utilement en complément de ce numéro consacré à la gouvernance multiniveau.

\section{Does Multi-Level Governance Reduce the Need for National Government?}

\author{
Par Marc Hooghe \\ European Political Science, vol. 11, n 1, \\ 2012, p. 90-95
}

Prenant comme point de départ l'absence de gouvernement en Belgique pendant plus d'un an suivant la réforme constitutionnelle de 2010, l'auteur de cet article s'interroge sur la nécessité d'un gouvernement national dans le contexte d'un système gouvernemental multiniveau. Il semble en effet que les autres ordres de gouvernement prennent le relais lorsqu'un niveau est défaillant. C'est d'autant plus perceptible en Belgique où l'Union européenne peut jouer un rôle dans la gouvernance du pays. L'un des intérêts d'un système multiniveau pourrait donc résider dans cette capacité à rattraper les défaillances et à servir de protection en cas de faillite d'un des niveaux.

\section{La comparaison de politiques publiques infranationales : méthodes et pratiques}

Revue internationale de politique comparée, vol. 19, n² 2, 2012, 174 p.

Deux journées d'étude sont à l'origine de ce numéro de la Revue internationale de politique comparée qui porte sur les défis de la méthodologie et de la comparaison de politiques publiques infranationales. En ressortent sept articles portant sur différents aspects de la recherche comparative avec pour sujets les politiques et les échelons de gouvernement infranationaux. Les grandes thématiques abordées par les auteurs sont notamment les régimes d'action publique, les politiques publiques infranationales et les différentes méthodes et stratégies de recherches comparatives.

\section{Multi-Level (Territorial) Governance: Three Criticisms}

\section{Par Andreas Faludi}

Planning, Theory \& Practice, vol. $13, n^{\circ} 2$, 2012, p. 197-211

Pour l'auteur de cet article, le concept de gouvernance territoriale multiniveau est en soi problématique pour trois raisons. D'abord, il considère que l'ajout de l'adjectif " territoriale » est redondant puisque la gouvernance est déjà circonscrite à un territoire donné. Ensuite, il estime que le concept est ambigu puisqu'il réfère généralement aux relations verticales entre les divers paliers d'un gouvernement et non à la gouvernance proprement dite. Enfin, l'auteur juge que la gouvernance multiniveau perçoit la gouvernance comme étant associée à un territoire physique plutôt que comme un construit social malléable et changeant.

\section{The Multi-Level Governance of Wales: Layer Cake or Marble Cake?}

Par Tom Entwistle, James Downe, Valeria Guarneros-Meza et Steve Martin The British Journal of Politics and International Relations, septembre 2012

Depuis la décentralisation mise en œuvre par le Royaume-Uni, la gouvernance du pays de Galles fonctionne selon quatre paliers : l'Union européenne, le RoyaumeUni, le national et le local. Selon les auteurs de cet article, la gouvernance multiniveau pourrait être comparée à un gâteau étagé, où chaque palier de gouvernement œuvre dans son propre champ de compétence, ou alors à un gâteau marbré, où les fonctions et les responsabilités de chacun sont entrelacées et se rejoignent. À la suite d'un sondage conduit auprès de cadres supérieurs de l'administration publique galloise, il semble que les deux types d'interprétation coexistent. 


\section{Adapting Federalism: Indigenous Multilevel Governance in Canada and the United States}

\author{
Par Martin Papillon \\ Publius: The Journal of Federalism, vol. 42, \\ $n^{\circ} 2,2012$, p. 289-312
}

Cette étude comparative s'intéresse à l'évolution des régimes fédéraux canadien et américain, plus particulièrement aux mécanismes d'adaptation mis en place pour répondre aux désirs d'autodétermination des populations autochtones. Faisant une revue de l'évolution des régimes, l'auteur suggère que les deux fédérations ont finalement eu le même type de parcours en instaurant une gouvernance multiniveau. Celle-ci leur aura permis d'ajouter différentes strates de gouvernance à la structure fédérale tout en préservant les fondations de cette dernière.

\section{On the Territoriality of Cross-Border Cooperation: "Institutional Mapping" in a Multi-Level Context}

\section{Par Tobias Chilla, Estelle Evrard et Christian Schulz \\ European Planning Studies, vol. 20, $n^{\circ} 6$, 2012, p. 961-980}

La territorialité est comprise comme le pouvoir d'exercer sa compétence sur un espace donné. La mondialisation de même que les structures supranationales bousculent peu à peu ces conventions spatiales vers une coopération transfrontalière. Cet article propose une cartographie des institutions transfrontalières en trois temps : en faisant la cartographie mutiniveau de ces institutions, en cartographiant les arènes politiques impliquées et en cartographiant la topographie politique. Prenant comme exemple le Luxembourg, les auteurs affirment que malgré le fait que l'instauration d'une territorialité transfrontalière ne peut remplacer la territorialité conventionnelle, elle pourrait voir émerger un système de territorialité multiniveau.

\section{Multi-Level Governance of Forest Resources}

Par Esther Mwangi et Andrew Wardell International Journal of the Commons, vol. 6, $n^{\circ} 2,2012$, p. 79-103

La gestion des ressources forestières nécessite différents niveaux d'implication des secteurs privé et public et de la société civile. C'est cette possibilité d'interaction que la gouvernance multiniveau semble offrir. Cet article définit la gouvernance multiniveau, explique en quoi celle-ci est pertinente et enfin décrit les processus et la structure de la gouvernance multiniveau d'un point de vue pratique. L'article insiste sur la nécessité de mettre en place ce type d'approche, plus complexe, qui tient compte du fonctionnement des politiques en tant qu'instruments de gouvernance et d'organisation des communautés au sein des systèmes de pouvoir et d'autorité.

\section{Multi-Level Governance of Regional Economic Development in Norway and Sweden: Too Much or Too Little Top-down Control?}

\section{Par Gro Sandkjaer Hanssen, Erik} Nergaard, Jon Pierre et Asgeir Skaalholt Urban Research \& Practice, vol. 4, $n^{\circ} 1$, 2011, p. 38-57

Le niveau régional a été ciblé comme étant un niveau institutionnel clé dans les politiques de développement économique. Or, en Norvège et en Suède, le développement économique régional se trouve limité par son éclatement, puisqu'il est soumis au contrôle des Parlements régionaux alors que les instruments les plus importants pour stimuler le développement économique régional sont contrôlés par le gouvernement national et des bureaux régionaux d'État. Dans cet article, les auteurs évaluent les effets escomptés de la centralisation et de la décentralisation des pouvoirs pour renforcer le développement économique régional. 